Théologiques

Théologiques

\title{
Du pantocrator au crucifié
}

\section{Jean Richard}

Volume 8, numéro 2, automne 2000

Le pouvoir

URI : https://id.erudit.org/iderudit/005025ar

DOI : https://doi.org/10.7202/005025ar

Aller au sommaire du numéro

\section{Éditeur(s)}

Faculté de théologie de l'Université de Montréal

\section{ISSN}

1188-7109 (imprimé)

1492-1413 (numérique)

Découvrir la revue

\section{Citer cet article}

Richard, J. (2000). Du pantocrator au crucifié. Théologiques, 8(2), 49-75. https://doi.org/10.7202/005025ar

\section{Résumé de l'article}

Sur la question des rapports entre le Pantocrator (le premier article desanciens Symboles chrétiens) et le Crucifié (le deuxième article), nousprésentons d'abord deux options bien différentes de théologienscontemporains : l'une qui souscrit à la thèse d'une « kénose " de Dieu (leDieu crucifié), l'autre qui la refuse. Nous proposons ensuite nos propresvues, en suivant la ligne de pensée de Hans Jonas dans son "Concept deDieu après Auschwitz ». Finalement, nous indiquons ce que la croyancechrétienne à la résurrection peut apporter à la thèse de Jonas et,réciproquement, comment la critique radicale de ce dernier peut aider àconcevoir une foi non supranaturaliste en la résurrection. d'utilisation que vous pouvez consulter en ligne.

https://apropos.erudit.org/fr/usagers/politique-dutilisation/ 
Théologiques 8/2 (2000) 49-75

\title{
Du pantocrator au crucifié
}

\author{
Jean RICHARD \\ Faculté de théologie et \\ de sciences religieuses \\ Université Laval \\ Québec
}

Ce titre qu'on m'a proposé, et que j'accepte volontiers, indique très bien ce dont il est question. Il s'agit du rapport entre le premier et le deuxième article du Credo chrétien, le rapport entre le Père tout-puissant et son Fils, le Christ crucifié. Il y a manifestement une tension entre ces deux pôles de la foi, une tension qui, en principe, se résout dans le troisième article, dans l'unité de l'Esprit Saint.

Selon cette vision de la foi chrétienne, le Credo représente beaucoup plus qu'une norme, qu'une simple mesure de l'orthodoxie; il exprime la dynamique même de la vie de foi, en tension constante entre la toute-puissance du Père et l'abaissement du Fils. Cette même tension explique les différents types de christianisme qui se sont succédés au cours de l'histoire, tout autant que les différentes sensibilités chrétiennes qui se manifestent et s'affrontent encore aujourd'hui parmi nous.

On pourrait dire à cet égard que, dans l'évolution de la foi chrétienne, l'accent est passé du pôle de la toute-puissance divine à celui de la souffrance du Crucifié. Dans les premiers siècles de l'Église, à l'époque des premiers grands conciles, le mouvement se faisait en sens inverse : du Crucifié au Pantocrator. Il s'agissait alors de faire valoir que le Fils était l'égal du Père, de même nature que le Père, c'est-à-dire de même puissance que lui. Mais le centre de la foi s'est manifestement déplacé, du premier au deuxième article du Credo. Aujourd'hui, la tendance va plutôt à penser que le Père est l'égal du Fils, c'est-à-dire qu'il assume son abaissement, qu'il est présent, souffrant et mourant avec lui et en lui sur la croix. 
Pour rendre compte adéquatement de l'état de la foi chrétienne, du sensus fidei, on doit ajouter cependant que la communauté de foi présente aujourd'hui un pluralisme tout aussi manifeste que celui de la société civile. C'est dire que les différents courants qui sont apparus au cours des âges se font encore sentir aujourd'hui au cœur de chaque communauté, au-delà même des limites confessionnelles, dans toutes les grandes Églises chrétiennes. Si l'on admet, au principe même de la foi, la tension dynamique dont nous avons parlé, on pourra reconnaître dans cette situation la grande richesse de l'Évangile.

Mais la reconnaissance d'un tel état de fait commande aussi le travail du théologien. Il devra prendre acte de ces différentes figures de la foi chrétienne aujourd'hui. Cela l'obligera à relativiser sa propre position, lui évitant par là de glisser dans le dogmatisme. Il ne devrait pas en rester là cependant. S'il veut faire plus que l'histoire de la pensée chrétienne aujourd'hui, en surplombant les différents courants chrétiens, s'il veut vraiment faire théologie, il devra lui-même s'engager, prendre option, se faire le porte-parole, la conscience réflexive d'un courant particulier qui lui a donné accès au coeur de l'Évangile ${ }^{1}$.

Commençons donc par faire état des deux courants opposés sur la question qui nous intéresse. Nous tenterons ensuite de clarifier la position qui est la nôtre.

1. J'avoue faire ici la théologie à la manière d'Ernst Troeltsch, tel qu'il l'exprimait dans son cours de 1912-1913 à Heidelberg: "Comme Schleiermacher, nous construisons nous aussi sur la conscience présente de la communauté. Pour une théologie de la conscience, il ne s'agit pas de faire un exposé du christianisme dans sa totalité, mais seulement de comprendre le christianisme d'aujourd'hui. Cela n'est pas facile ; ce n'est possible qu'avec courage et avec une prise de position personnelle. Quiconque entreprend cette tâche en assume la responsabilité. La seule preuve possible est celle de sa théologie, car il n'y en a pas d'autre que le sentiment qu'ici se trouve le grand et puissant courant qui emporte le présent et le conduit vers l'avenir. À ce point précis, cesse la stricte science démonstrative ; le sentiment personnel, la prise de position pratique constituent l'essentiel » (Glaubenslehre [1925], Scientia Verlag Aalen, 1981, p. 14). 


\section{Le point de vue de la kénose de Dieu}

Voyons d'abord un témoin du courant qui domine aujourd'hui la pensée chrétienne, celui de l'abaissement (kénose) de Dieu. Il s'agit d'un article de Jean-Marie Glé, jésuite, paru récemment dans la revue Christus. L'auteur se présente comme suit : "j'appartiens à l'Église catholique, à une congrégation religieuse, à une communauté, au corps professoral d'une faculté de théologie catholique ${ }^{2}$ ", en l'occurrence, le Centre Sèvres de Paris. L'article porte sur les difficultés que ressentent aujourd'hui les croyants de "penser Dieu créateur ». Il s'agit donc du premier article du Credo et de la nécessité de repenser aujourd'hui la foi en Dieu créateur. La difficulté vient justement de l'association de l'idée de création à celle du Père tout-puissant.

Plus précisément, deux traits caractéristiques de la situation contemporaine s'opposent à cette idée d'une toute-puissance créatrice. Et l'on se trouve par là déjà en plein paradoxe, puisque ces deux traits sont, d'une part, la pleine autonomie humaine revendiquée par la modernité et, d'autre part, l'énorme souffrance qu'a connue l'humanité au cours du $\mathrm{XX}^{\mathrm{e}}$ siècle.

Il importe de bien saisir le sens de cette autonomie moderne. On doit dire qu'il s'agit là d'abord d'un fait socio-culturel : un fait d'évolution culturelle tout aussi naturel et normal que celui de l'enfant qui, devenu adulte, acquiert son autonomie face à sa famille. Celle-ci, jusque là, l'avait encadré, dirigé ; elle lui laisse maintenant sa liberté, ce qui rendra possible la constitution de nouveaux liens avec les parents. Il en va de même dans l'évolution de l'humanité :

Au sein de la société moderne occidentale a émergé l'individu : l'appartenance à une famille, à un milieu social, à une région ou à un pays, ne détermine plus le comportement de la personne. La mondialisation va de pair avec une individualisation toujours plus forte au sein de la société. Celle-ci n'est pas configurée comme une totalité dont les individus seraient des parties, mais comme un ensemble d'individus qui sont chacun un centre de décision autonome ${ }^{3}$.

2. Jean-Marie GLé, "Penser Dieu créateur. Une démarche difficile ", Christus, $\mathrm{n}^{\mathrm{O}} 185$ (janvier 2000), p. 43.

3. Ibid., p. 43. 
Une telle révolution culturelle ne signifie pas le refus de toute autorité ; elle implique cependant une transformation radicale de la notion d'autorité et de son exercice. Cela s'applique à tous les ordres d'autorité, jusqu'au niveau théologique. Ainsi, la modernité n'est pas intrinsèquement athée ; elle commande cependant une profonde transformation de la pensée religieuse quant aux rapports de l'humanité avec Dieu son créateur. Or, loin d'être démunie face à ces nouvelles exigences des temps modernes, la pensée chrétienne contient, en son centre même, les ressources nécessaires pour y répondre. Tel est justement le sens nouveau qu'inspire aujourd'hui la pensée de la croix : "Au pied de la croix, je peux découvrir que Dieu, dans la figure du Père, n'est pas un Dieu qui s'impose, qui intervient dans l'histoire humaine ${ }^{4}$ ». Dieu nous laisse libres ; plus encore il nous remet la pleine responsabilité de construire le monde, de poursuivre l'oeuvre de la création.

Nous venons de décrire l'autonomie dans sa face lumineuse, dans son essence même. Mais il y a aussi un aspect plus obscur de cette même réalité, telle qu'elle se présente concrètement, historiquement, au cours du dernier siècle tout spécialement. Jean-Marie Glé ne perd pas de vue cette face obscure de la modernité récente :

Pour autant, je n'oublie pas la réalité historique des camps et la question qu'elle pose à l'égard d'une humanité capable de tant d'horreurs. Je n'oublie pas non plus que la toute-puissance est aussi du côté de l'être humain moderne, qui revendique le pouvoir de la science et de la technique sur une nature "mécanisée ». La toute-puissance techno-scientifique, qui permet Auschwitz et les autres atrocités que le $\mathrm{XX}^{\mathrm{e}}$ siècle a connues, se retourne en son contraire : l'impuissance de l'humanité à faire face à l'automate « totalitaire ». Dieu est touché, mais l'humanité aussi ${ }^{5}$.

On ne peut donc plus parler de Dieu simplement à partir de la nature essentielle des choses, à partir de " la différence qualitative infinie " qui le distingue de l'humanité. On ne peut faire abstraction de la situation historique, existentielle, qui est la nôtre : "Quand il essaie de penser la création, un Européen du $\mathrm{XX}^{\mathrm{e}}$ siècle ne peut s'abstraire du contexte dans lequel il réfléchit. L'humanité souffrante est son horizon, l'humanité qui ressent concrètement la situation malheu-

4. Ibid., p. 41.

5. Ibid., p. 42. 
reuse dans laquelle elle est ${ }^{6}{ }$. Or c'est précisément cette situation qui nous rend si difficile la pensée de la toute-puissance : "Au nom de "Dieu créateur" est associée l'idée de la "toute-puissance" divine, qui est de plus en plus difficile à penser pour nos contemporains ; elle est même insoutenable et impensable pour beaucoup ${ }^{7}$ ».

Ce n'est pas le comportement de Dieu à travers l'histoire du salut qui rend si difficile cette idée de toute-puissance. On n'y gagnera pas beaucoup, par conséquent, à insister sur le fait qu'il s'agit d'une toutepuissance libératrice, d'une toute-puissance d'amour. Là n'est pas le problème. Il réside plutôt dans la situation humaine en cette période de l'histoire : dans le fait que la puissance humaine - puissance scientifique, technologique, économique - n'a jamais été si grande, que ses développements à venir paraissent illimités, mais que ces perspectives, loin d'être source d'espérance, nous font au contraire craindre le pire, d'après l'expérience que nous en avons fait au cours du siècle qui vient de s'achever.

La solution - le salut - ne peut dès lors consister dans l'évocation d'une puissance plus grande encore que tout ce que peut imaginer l'esprit humain (Anselme) : en somme, une toute-puissance divine. Car le problème ne vient pas d'un manque de puissance, mais de sa perversion. La solution devra donc être la conversion de la puissance, non pas son augmentation, ni sa simple suppression. Or c'est là tout l'enseignement de l'Évangile jusqu'à son point culminant, le Golgotha. C'est là le point fort de l'enseignement de Jésus à ses disciples : la conversion du pouvoir en service; le plus grand qui doit se mettre au service du plus petit. Et Jésus lui-même, le Maître qui parle avec autorité, qui agit avec puissance, ne vient pas pour être servi mais pour servir et donner sa vie par amour. Jusqu'ici tout chrétien sera bien d'accord. Les avis divergent cependant quant à la signification de tout cela pour la puissance divine. La position que nous présentons maintenant soutient que cela s'applique aussi à la toute-puissance divine, que cela signifie précisément la conversion, le renversement de notre affirmation de la toute-puissance. Dans le Christ crucifié, Dieu renonce à sa toute-puissance ; il donne lui-même sa vie par amour, pour le salut du monde.

6. Ibid., p. 39.

7. Ibid., p. 39-40. 
Ces dernières réflexions débordent le texte même de Jean-Marie Glé ; elles prétendent cependant en exprimer le sens profond. Avant de passer à autre chose, je tiens encore à signaler la référence répétée que fait l'auteur aux lettres de prison de Dietrich Bonhoeffer. Car Bonhoeffer n'est pas un simple théologien parmi d'autres. C'est, au sens le plus strict, un témoin (martyr), situé au coeur de la situation, qui fait pleinement l'expérience des ténèbres de son époque. C'est de là qu'il voit l'humanité face à Dieu et qu'il en parle. On comprend mieux alors un paradoxe qui en étonne beaucoup. D'abord, son affirmation de la pleine autonomie séculière de l'homme moderne : "En devenant majeur, nous sommes amenés à reconnaître de façon plus vraie notre situation devant Dieu. Dieu nous fait savoir qu'il nous faut vivre en tant qu'hommes qui parviennent à vivre sans Dieu ${ }^{8}$ ". Mais cette même lettre du 16 juillet 1944 se termine par un rappel de la misère humaine. Dieu est reconnu alors, non plus simplement comme abandonnant l'homme à ses responsabilités, mais comme celui qui souffre avec lui : "Dieu se laisse déloger du monde et clouer sur la croix. Dieu est impuissant et faible dans le monde et ainsi seulement il est avec nous et nous aide. Matthieu 8,17 indique clairement que le Christ ne nous aide pas par sa toute-puissance, mais par sa faiblesse et ses souffrances ${ }^{9} »$.

\section{Le point de vue de la toute-puissance de Dieu}

Passons maintenant à l'autre courant de la pensée chrétienne contemporaine, où l'idée de la toute-puissance divine est maintenue dans son intégrité. J'ai choisi comme porte-parole de cet autre sensus fidei un autre théologien de Paris, Jean-Pierre Batut, relié cette fois au studium du séminaire de Paris. Je m'arrêterai plus spécialement à son article paru récemment dans la revue Communio sous le titre : «Dieu le Père tout-puissant ». J'insiste sur le fait qu'on trouve là l'expression d'une autre sensibilité chrétienne et théologique. La différence entre ces deux types de théologie ne vient donc pas de ce que l'une serait plus

8. Dietrich BONHOEFFER, «Lettre du 16 juillet 1944.»In : Résistance et soumission. Lettres et notes de captivité, nouvelle édition, Genève, Labor et Fides, 1973, p. 366.

9. $\quad$ Ibid., p. 367. 
critique, l'autre plus naïve. Le texte que je présente maintenant n'est pas moins critique que le précédent, tout au contraire.

L'auteur entend, en effet, rétablir le sens biblique et chrétien de la toute-puissance. Celle-ci n'est pas le privilège de quelqu'un qui peut faire tout ce qu'il veut. Tout l'article vise à dépasser cette notion si profondément ancrée dans la conscience religieuse, notion d'où surgit spontanément la question, et le scandale : "Si Dieu est tout-puissant, pourquoi le mal existe-t-il ? " Car si Dieu est tout-puissant, il peut faire tout ce qu'il veut et, par conséquent, empêcher tout ce qu'il ne veut pas. Or le bon Dieu ne peut vouloir que le bien.

Pour remettre les choses en place, Batut va s'attaquer d'abord au mot lui-même "tout-puissant». Ce terme rend plus ou moins bien le grec "Pantocrator", qui se retrouve dans les premiers Symboles de foi, dont le Symbole de Nicée-Constantinople. Car, à la différence du latin «omnipotens» et du français «tout-puissant», le titre de "Pantocrator» est un terme relationnel, qui désigne "un rapport permanent de Dieu à l'univers ", et qu'on pourrait traduire littéralement : " qui tient ensemble toutes choses ${ }^{10}$ ». Ainsi, " on est pantocrator sur quelque chose, alors qu'on est omnipotens, tout-puissant, en soi ». D'où le glissement de sens : "Alors que le Pantocrator était celui qui règne sur le tout, l'Omnipotens pourra être vu comme celui qui peut tout faire, le détenteur jupitérien d'un pouvoir absolu ${ }^{11}$ ».

Ces considérations étymologiques ne sont encore qu'une entrée en matière. Batut en arrive vraiment à la chose (ad rem) quand il en vient à parler du contexte et de la signification biblique du terme "Pantocrator». Une autre précision s'impose alors. Ce titre ne désigne pas seulement la seigneurie universelle de Dieu sur le monde, une domination qui s'exercerait de façon identique en tout temps. Selon la révélation biblique, la toute-puissance divine comporte de fortes connotations historiques : " pour l'Écriture, la puissance de Dieu s'exerce toujours (même dans les récits de création) à l'intérieur d'un cadre temporel, historique ${ }^{12}{ }^{\prime}$, qui est celui de l'histoire du salut. En

10. Jean-Pierre BATUT, " Dieu le Père tout-puissant : Réflexion à propos d'un mot litigieux ", Communio, XXIII/6-XXIV/1 (novembre 1998-février 1999), p. 59.

11. Ibid., p. 68.

12. Ibid., p. 60. 
somme, l'affirmation de principe "Dieu règne sur le monde " signifie concrètement qu'il établit son règne sur le monde au cours de l'histoire : "De la même façon que le Christ, qui est le Fils, le devient très réellement au terme d'une existence orientée vers le sacrifice pascal, de même le Père, qui est le Tout-puissant, prendra possession à la fin des temps d'une souveraineté sur le monde qu'il n'a à vrai dire jamais cessé d'exercer ${ }^{13}$ ". Cela se vérifie de façon particulièrement manifeste dans le livre de l'Apocalypse, où se retrouve la quasi-totalité des mentions du Pantocrator. Par exemple : "Nous te rendons grâce, Seigneur Dieu Pantocrator, qui es et qui étais, car tu as saisi ta grande puissance et établi ton règne » (Ap 11, 17). Batut commente ici bien justement : « il s'agit du constat paradoxal par le voyant de la présence déjà effective d'une réalité encore à venir ${ }^{14}$ ».

Or cette dimension historique, que présentent les mentions bibliques de la toute-puissance divine, implique elle-même une connotation relationnelle, cette fois en un sens plus profond, spécifiquement interpersonnel. On rendrait bien la pensée de l'auteur, il me semble, en parlant ici d'une histoire d'alliance du Dieu-Père avec son partenaire humain. Ainsi, corrélativement à l'histoire du salut, " la Bible met en lumière le rôle décisif de la réponse de l'homme, car l'histoire est le lieu de l'émergence et de la réponse des libertés. Celles-ci ont le redoutable pouvoir d'infléchir le dessein de Dieu, sans pour autant le détourner de son orientation originelle ${ }^{15} »$.

C'est dire que, à la fin de l'histoire, triomphera la toute-puissance du Pantocrator. Mais cela s'accomplit toujours dans le cadre de l'alliance, sans violence, dans le respect total de la liberté humaine. Ce qui signifie une corrélation parfaite entre, d'une part, la toute-puissance du Pantocrator et, d'autre part, la soumission spontanée des humains sous forme d'obéissance filiale. Et voilà justement ce qui se trouve en même temps réalisé et annoncé dans la vie et la mort du Christ, le parfait partenaire de l'alliance, le Fils par excellence. C'est donc en lui, avec lui et par lui que le Père tout-puissant réalise son règne en notre monde :

13. Ibid., p. 61.

14. Ibid., p. 60.

15. Ibid., p. 60. 
Encore faut-il [pour cela] qu'une fidélité sans faille réponde enfin, du côté de l'homme, à la fidélité de Dieu. Tel est l'enjeu de l'incarnation. En Jésus, une liberté humaine [...] a donné enfin une adhésion totale à l'initiative du Père. Ce faisant, cette existence humaine s'est trouvée "filialisée" de part en part, et ce jusque dans l'acte de mourir en remettant son esprit entre les mains du Père ${ }^{16}$.

Mais il faut alors pousser plus avant encore l'idée d'une toute-puissance « relationnelle ». La toute-puissance divine se trouve ainsi dite non seulement en raison de sa relation créatrice à l'univers, et non seulement en raison de sa relation d'alliance avec nos libertés créées, mais plus encore à cause de la communication que fait Dieu de sa propre puissance à tous ceux et celles qu'il suscite lui-même comme partenaires de son alliance. Voilà, encore une fois, ce qui apparaît de façon manifeste dans la destinée du Fils : "Ce titre de "Seigneur" est privilégié par l'Écriture pour indiquer la transmission au Christ de la souveraineté du Père sur tous les "étages" de l'univers ${ }^{17}$ ". Or cette autocommunication de la puissance divine s'accomplit principiellement, de tout éternité, "dans l'engendrement du Fils et la spiration de l'Esprit ». Mais elle se réalise ultimement dans "l'événement historique de la résurrection, dans lequel le Christ est constitué "Seigneur” par le Père ${ }^{18} »$.

Entre l'origine éternelle et ce terme ultime que représente la résurrection du Christ, prend place maintenant toute l'histoire de la création et du salut de l'humanité. Il y a là deux aspects bien distincts à considérer : la constitution même de la liberté humaine par l'acte créateur et l'histoire mouvementée de l'alliance divino-humaine. Dans les deux cas, de façon bien différente cependant, la toute-puissance divine apparaît comme relationnelle à la liberté humaine, dans un acte d'autocommunication.

Pour ce qui concerne le premier aspect, Jean-Pierre Batut montre bien comment la toute-puissance divine, loin d'être une entrave à la liberté humaine, en constitue au contraire l'origine et le fondement. En effet, il en va tout autrement de la puissance infinie de Dieu et de la puissance toute limitée qui est la nôtre. Notre puissance, notre pou-

16. Ibid., p. 60-61.

17. Ibid., p. 59.

18. Ibid., p. 58 et 73 . 
voir d'être est constamment menacé ; nous devons le retenir jalousement, nous retrouvant ainsi dans une position conflictuelle face aux autres libertés qui nous affrontent. Mais cela ne s'applique pas à la toute-puissance divine : "Alors que nous-mêmes passons notre temps à redouter que ce que nous avons ne nous échappe, et qu'un autre le prenne, Dieu ne peut en aucune manière redouter d'être dépossédé de quoi que ce soit par les hommes ${ }^{19}$ ". Au contraire, la puissance infinie de Dieu est source de toute liberté humaine, comme de toute autonomie créée, puisque celles-ci ne surgissent que du fait de leur participation à cette même toute-puissance divine : "Parce qu'il est sans l'ombre d'un "faux rapport à l'égard de celui qu'il veut rendre libre”, le Tout-Puissant est en mesure de susciter d'authentiques libertés ${ }^{20} »$.

Batut se réfère ici à un passage du Journal de Kierkegaard, en 1846 (Papirer, VII A 181), passage qui se termine par cette phrase remarquable : "Seule l'omnipotence peut se récupérer alors qu'elle se donne, et ce rapport constitue précisément l'indépendance de celui qui reçoit. " On trouve là, bien affirmée, l'idée que l'autonomie (ou la liberté) humaine est un effet, un don de la toute-puissance divine, qu'elle provient d'une autocommunication divine. Mais on pourrait y voir plus que cela, surtout si l'on tient compte de l'inspiration luthérienne omniprésente chez Kierkegaard. Le don que la toute-puissance fait d'elle-même pourrait être interprété comme un abandon, comme un renoncement à cette même toute-puissance. En somme, cela pourrait signifier une kénose divine, le fait que Dieu se dépouille de ce qui constitue la marque spécifique de la divinité, la toute-puissance précisément. L'affirmation que "seule l'omnipotence peut se récupérer alors qu'elle se donne » signifierait alors le paradoxe d'un Dieu qui demeure Dieu tout en se dépouillant de sa divinité, le paradoxe d'un Dieu crucifié, en d'autres termes, le paradoxe d'une toute-puissance souffrante. Une telle interprétation gagne encore en vraisemblance si l'on tient compte non seulement du fait même de l'autonomie créée, mais aussi de tous les aléas de la liberté humaine au cours de l'histoire. Celle-ci a, en effet, "le redoutable pouvoir d'infléchir le dessein de Dieu ", comme on pouvait lire plus haut, ce qui n'est pas sans assombrir de façon tragique l'alliance divino-humaine. Quoiqu'il en soit,

19. Ibid., p. 72.

20. Ibid., p. 73. 
Batut oppose ici une fin de non-recevoir. Il refuse expressément de s'engager dans cette voie :

Sous l'influence de pensées suggestives, mais sujettes à caution, on a parfois conclu de l'existence de cette liberté à la nécessité de renoncer à l'idée même de toute-puissance, transposant ainsi sur la personne du Père la théologie de la "kénose » que la tradition chrétienne, à la suite de saint Paul, à développée pour rendre compte de l'abaissement du Christ. Faut-il alors imaginer que, pour nous rendre libres, le Père doive devenir "non-puissant ", en laissant sa puissance s'abîmer tout entière dans le gouffre de la contingence ?21

Sans doute, faudrait-il apporter quelques précisions pour reconstituer la thèse ici incriminée. D'abord, ce n'est pas l'existence même de la liberté humaine qui fait conclure à la kénose de Dieu, mais bien plutôt l'histoire de ses aliénations par rapport à son fondement divin, la douloureuse histoire du "péché du monde ». Ensuite, ce qu'affirme cette thèse, c'est que Dieu lui-même, par amour, renonce à sa toute-puissance pour tout le temps de l'histoire ; ce n'est pas nous qui la lui refusons. Enfin, précisément parce que ce renoncement, cet abaissement, vient de Dieu, il devient lui-même expression et signe de sa toute-puissance. C'est ainsi qu'on peut comprendre le mot de Kierkegaard : "Seule l'omnipotence peut se récupérer alors qu'elle se donne. »

Mais, même admises toutes ces précisions, il est peu probable que Jean-Pierre Batut — et tout le courant théologique qu'il représente puisse changer d'idée et admettre la pensée d'une kénose de Dieu. Cela peut surprendre, car il semble bien que cette thèse ne soit que l'ultime aboutissement, la conclusion finale des réflexions développées par notre auteur. Pourquoi alors refuser de poser ce dernier pas, de franchir cette ultime étape ? Mais cette étape présente ceci de particulier, qu'avec elle la pensée bascule dans le paradoxe. Or, pour une théologie ultra-catholique, toute pensée du paradoxe, spécialement celle du paradoxe paulino-luthérien, peut paraître suspecte, "sujette à caution ». Une telle explication, cependant, ne me semble pas suffisante ; elle ne va pas au fond des choses. Elle ne fait pas voir, en effet, toute la distance qui sépare les deux courants de pensée, en suggérant simplement que l'un est plus traditionaliste, l'autre plus progressif, poussant plus avant dans la même ligne.

21. Ibid., p. 71-72. 
À mon avis, la différence est beaucoup plus profonde. C'est qu'on a, d'une part, une théologie qui se maintient au niveau de la pensée, qui ne considère que l'évolution de la pensée chrétienne, qui n'examine tout au plus que l'histoire biblique, l'histoire du salut; et, d'autre part, une théologie qui tient compte aussi de la situation historique, tout spécialement la situation présente. Pour mieux saisir de quoi il s'agit, il sera utile de rappeler ici les premières lignes de la Théologie systématique de Paul Tillich, qui distingue bien les deux pôles donnant lieu à deux types bien différents de théologie : "Un système théologique doit répondre à deux besoins fondamentaux : l'énoncé de la vérité du message chrétien et l'interprétation de cette vérité pour chaque génération nouvelle. La théologie oscille entre deux pôles : l'éternelle vérité de son fondement et la situation temporelle dans laquelle la vérité éternelle doit être reçue ${ }^{22} »$.

Manifestement, Batut met tout l'effort de sa recherche et de sa réflexion sur le pôle du message chrétien. Il excelle en ce domaine. Il y fait preuve d'une grande érudition et d'un esprit critique qui n'est pas sans audace, tout spécialement quand il recommande là-dessus un retour à la théologie anténicéeene, telle qu'illustrée dans le Traité des principes d'Origène. Par contre, notre auteur ne tient aucun compte de la situation particulière qui, à telle ou telle époque, pourrait influer sur la pensée chrétienne en lui conférant une orientation nouvelle. Aussi, au début de l'article, soulève-t-il la question du mal. Mais il s'agit du mal en général, en tant que caractéristique permanente de la condition humaine. De même, vers la fin de l'étude, sera posée la question de la liberté humaine face à la toute-puissance divine. Mais, là encore, il est question de la liberté en général, non pas de l'autonomie caractéristique de la modernité.

On comprend dès lors la forte réaction de Batut contre la vision de Hans Jonas ${ }^{23}$. Ce dernier, en effet, met lui-même l'accent sur la situation, en proposant une théologie après Auschwitz. Sa conférence, Le concept de Dieu après Auschwitz est devenue un classique de la théologie négative de la toute-puissance, au même titre que les Lettres de

22. Paul Tillich, Systematic Theology, Vol. I, The University of Chicago Press, 1951, p. 3.

23. J.-P. BATUT, « Dieu le Père tout-puissant », p. 71, note 21, et p. 73. 
captivité de Bonhoeffer. C'est de ce côté que nous nous orientons maintenant.

\section{La théologie après Auschwitz}

Dans sa dernière mouture, ce texte de Jonas est celui d'une conférence prononcée à l'Université de Tübingen en 1984, et qu'il dédie à toutes les victimes de la Shoah, dont sa mère, elle-même morte à Auschwitz : "J'ai cru devoir ne pas refuser à ces ombres quelque chose comme une réponse à leur cri, depuis longtemps expiré, vers un Dieu muet ${ }^{24}$ ».

Tout comme dans l'article de Batut, le point de départ est ici la question de Job : qu'en est-il de la toute-puissance divine face au mal qui sévit dans le monde ? Mais il ne s'agit plus de la question du mal en général. Jonas, en effet, réclame le droit « de laisser la violence d'une expérience unique et monstrueuse intervenir dans les interrogations sur ce qu'il en est de $\operatorname{Dieu}^{25}$ ». Cette violence unique, que bien des Juifs appellent « l'horreur absolue », Jonas la décrit plutôt comme le « nonsens absolu », en faisant ressortir la parfaite indifférence qui régnait alors quant à toutes les valeurs pouvant éclairer la situation d'une quelconque lumière divine : "Ici ne trouvèrent place ni la fidélité ni l'infidélité, ni la foi ni l'incroyance, ni la faute ni son châtiment, ni l'épreuve, ni le témoignage, ni l'espoir de rédemption, pas même la force ou la faiblesse, l'héroïsme ou la lâcheté, le défi ou la soumission. Non, de tout cela Auschwitz, qui dévora même les enfants, n'a rien su ${ }^{26}$ ».

Auschwitz représente donc l'obscurité absolue, l'éclipse totale, le complet silence de Dieu. Pour Jonas, cependant, cela ne signifie pas la mort de Dieu : "Et quand on ne veut pas se séparer du concept de Dieu - comme le philosophe lui-même en a le droit —, on est obligé, pour ne pas l'abandonner, de le repenser à neuf et de chercher une réponse, neuve elle aussi, à la vielle question de $\mathrm{Job}^{27}$ ». Le sens du projet de Jonas apparaît ici bien clairement. Devant le caractère radical de sa vision, plusieurs penseront qu'il ne reste plus rien de la foi

24. Hans Jonas, Le Concept de Dieu après Auschwitz. Une voix juive, Paris, Payot \& Rivages, 1994, p. 8.

25. Ibid., p. 10.

26. Ibid., p. 11.

27. Ibid., p. 13. 
biblique, que le Dieu des pères se trouve défiguré, méconnaissable. Pourtant, s'il propose cette nouvelle vision, s'il donne congé au "Seigneur de l'histoire » et au Pantocrator, c'est précisément pour que la foi soit encore possible en ce temps du silence de Dieu, et pour qu'elle puisse aujourd'hui encore s'exprimer dans une certaine conceptualité théologique.

Mais une autre surprise nous attend encore ici. Car ce n'est pas d'abord sous le mode conceptuel mais sous la forme du mythe que Jonas va proposer sa nouvelle théologie. Il s'en explique lui-même en se référant à Platon qui autorisait " ce type de conjecture imagée [...] pour la sphère située au-delà de la connaissance ${ }^{28} »$. L'utilisation de la forme mythique rend donc pleinement manifeste le caractère symbolique de tout langage sur Dieu, même le plus conceptuel. Dans son commentaire, Catherine Chalier note une autre influence, au-delà de Platon, celle du gnosticisme, auquel Jonas a consacré ses premiers travaux. Car « les gnostiques recourent au mythe pour parler de l'état présent des choses - la terrible énigme de la souffrance humaine face à la bonté de Dieu. Ils élaborent divers récits qui rendent compte de cette contradiction insupportable, récits remontant à un drame survenu aux origines et expliquant pourquoi ce Dieu ne peut rien pour les hommes ${ }^{29}$ ». Ainsi, " à l'instar des gnostiques, mais dans une perspective juive, monothéiste donc, il tente, à son tour d'expliquer la réalité dramatique des jours sur lesquels nulle Providence ne veille, en imaginant ce qui advint aux temps des origines. Comme si, pour tenter de percer l'énigme d'un présent ténébreux et interdit de toute miséricorde, il fallait remonter au plus lointain jadis, à la geste créatrice ellemême $^{30}$ ». Voici donc le récit mythique que Jonas propose à notre réflexion :

Au commencement, par un choix insondable, le fond divin de l'Être décida de se livrer au hasard, au risque, à la diversité infinie du devenir. Et cela entièrement : la divinité, engagée dans l'aventure de l'espace et du temps, ne voulut rien retenir de soi ; il ne subsiste d'elle aucune partie préservée, immunisée, en état de diriger, de corriger, finalement de ga-

28. Ibid., p. 14.

29. Catherine CHALIER, "Dieu sans puissance", dans H. JONAS, Le Concept de Dieu après Auschwitz, p. 47-48.

30. Ibid., p. 51-52. 
rantir depuis l'au-delà l'oblique formation de son destin au sein de la création. L'esprit moderne repose sur cette immanence absolue ${ }^{31}$.

Dieu, pour que le monde soit et qu'il existe de par lui-même, a renoncé à son Être propre ; il s'est dépouillé de sa divinité, afin d'obtenir celleci, en retour, de l'odyssée des temps, donc chargée de la récolte fortuite d'une imprévisible expérience temporelle, lui-même, Dieu, étant alors transfiguré, ou peut-être aussi défiguré par elle. Dans un tel abandon de l'intégrité divine au profit du devenir sans restriction ne peut être admise aucune connaissance préalable, si ce n'est celle des possibilités qu'accorde l'être cosmique à travers ses propres conditions : ce sont justement les conditions auxquelles Dieu a livré sa cause, puisqu'il s'est dépouillé en faveur du monde ${ }^{32}$.

Dans son Introduction à Hans Jonas, Franz Josef Wetz note les deux motifs d'une telle conception théologique : c'est d'abord la prise en compte de la vision scientifique du monde, mais c'est aussi l'expérience d'Auschwitz ${ }^{33}$. La vision scientifique s'oppose à toute conception supranaturaliste, à toute intervention d'en haut dans le processus de l'évolution naturelle. Tel est le sens de l'affirmation de Jonas : "L'esprit moderne repose sur cette immanence absolue. » L'univers va son cours, selon ses propres lois, et selon toutes les contingences et hasards qu'elles impliquent. Donc pleine autonomie de tous les processus naturels.

Mais cela signifie aussi la pleine autonomie de la liberté humaine, une autonomie qui implique une puissance réelle, distincte de celle de Dieu : " Donc ce sur quoi la puissance [divine] agit doit avoir une puissance intrinsèque même si cette dernière provient de la première, et fut originairement dispensée à son détenteur en même temps que l'existence par un renoncement à soi de la puissance illimitée, cela dans l'acte de création justement ${ }^{34}$ ».

Une telle limitation de la puissance divine, du fait même de la création, est loin d'aller de soi dans une perspective biblique, puisqu'elle implique la négation de la toute-puissance. Il y a donc rupture mani-

31. H. Jonas, Le Concept de Dieu après Auschwitz, P. 14.

32. Ibid., p. 15-16.

33. Franz Josef WeTZ, Hans Jonas zur Einführung, Hamburg, Junius, 1994, p. 179-180.

34. H. JONAS, Le Concept de Dieu après Auschwitz, p. 30. 
feste par rapport aux récits bibliques de la création. Jonas ne s'en cache pas, d'autant moins qu'il ose lui-même proposer un nouveau mythe de la création, plus conforme à la vision moderne du monde. Mais il tient tout autant à affirmer la continuité avec la foi juive à propos de la création. Voilà pourquoi il insiste sur l'idée d'une auto-limitation divine, d'un auto-renoncement de Dieu à sa toute-puissance. Il peut alors se féliciter de rejoindre un courant de la tradition juive bien antérieur à la modernité : " mon mythe ne fait au fond que radicaliser l'idée du Tsimtsoum, ce concept cosmogonique central de la Cabale lurianique. Tsimtsoum veut dire contraction, retrait, autolimitation. Pour faire place au monde, le En-Sof du commencement, l'infini, a dû se contracter sur lui-même et laisser naître ainsi à l'extérieur de lui le vide, le néant, au sein duquel et à partir duquel il a pu créer le monde ${ }^{35} »$.

Nous n'avons considéré jusqu'ici que le premier aspect de la thèse de Jonas : la prise en compte de la vision scientifique du monde, de la pleine autonomie humaine et de la totale immanence de Dieu qu'elle commande. Voyons maintenant l'autre aspect, qui fait mieux voir encore le caractère radical de cette théologie : l'expérience d'Auschwitz. Nous touchons ici au cœur même de la thèse de Jonas. Ce fut annoncé dès le début par une question plus d'une fois reprise comme un leitmotiv : "Et Dieu laissa faire. Quel est ce Dieu qui a pu laisser faire ? [...] Quel Dieu a pu laisser faire cela ? ${ }^{36}{ }^{»}$

Vers la fin de la conférence, la question est reprise et la réponse est donnée en des termes aussi clairs que percutants. L'autolimitation divine est totale. Ce qui signifie qu'elle est sans retour. Ce n'est pas comme si Dieu retenait sa puissance et se refusait d'intervenir, à la façon des parents qui laissent leurs enfants prendre certains risques. En somme, c'est toute la question de la "permission divine » qui se trouve soulevée ici. Jusqu'à nos jours, telle fut la réponse de la théodicée : Dieu ne veut pas le mal, mais il le permet, pour un plus grand bien, ou pour éviter un plus grand mal. Mais cette réponse s'avère manifestement insuffisante devant "l'horreur absolue ». Dieu a-t-il permis Auschwitz ? Jonas répond lui-même sans détour :

35. Ibid., p. 37-38.

36. Ibid., p. 12 et 13 . 
Pendant toutes les années qu'a duré la furie d'Auschwitz, Dieu s'est tu. [...] Et moi, je dis maintenant : s'il n'est pas intervenu, ce n'est point qu'il ne le voulait pas, mais parce qu'il ne le pouvait pas. Je propose, pour des raisons inspirées par l'expérience contemporaine de façon déterminante, l'idée d'un Dieu qui pour un temps - le temps que dure le processus continué du monde - s'est dépouillé de tout pouvoir d'immixtion dans le cours physique des choses de ce monde; d'un Dieu qui donc répond au choc des événements mondains contre son être propre, non pas "d'une main forte et d'un bras tendu » - comme nous le récitons tous les ans, nous les Juifs, pour commémorer la sortie d'Égypte - mais en poursuivant son but inaccompli avec un mutisme pénétrant ${ }^{37}$.

La négation de la toute-puissance divine apparaît clairement ici dans toute sa signification : Dieu s'est dépouillé de tout pouvoir d'intervention dans l'histoire des peuples comme dans la vie des individus. Deux questions se posent alors, qu'il nous faut considérer attentivement. Une telle conception est-elle encore croyante ? Et puis, cette interprétation n'est-elle pas déjà chrétienne ?

La première question a été soulevée par Franz Josef Wetz, qui interprète le nouveau mythe de Jonas dans le sens de la mort de Dieu : " un Dieu ainsi livré au monde est vraiment un Dieu mort ${ }^{38}$ ". Wetz cite alors Lactance, Cicéron et Ambroise, pour montrer qu'un Dieu qui n'est plus secourable, un Dieu qui n'a plus souci du monde, est un Dieu qui n'a plus rien de divin, qui a perdu toute divinité. Et il conclut à propos de Jonas : «Un Dieu qui ne soutient plus le monde, l'histoire et les humains de façon secourable, mais qui se livre et s'abandonne, est pratiquement un Dieu mort ${ }^{39}$ ».

Une telle interprétation constitue manifestement une totale incompréhension. Pour commencer, elle contredit une affirmation explicite de Jonas, qui propose " le concept d'un dieu soucieux — non pas éloigné, détaché, en lui-même enfermé, mais au contraire impliqué dans ce dont il a le souci ${ }^{40}$ ". D'ailleurs, Hans Jonas a lui-même posé la question de façon existentielle, en demandant si, dans le cadre d'une telle conception, la foi pouvait se maintenir, la religion demeurer vivante : "Totale

37. Ibid., p. 34-35.

38. F.J. WETZ, Hans Jonas zur Einführung, p. 178.

39. Ibid., p. 179.

40. H. JONAS, Le Concept de Dieu après Auschwitz, p. 26. 
devient la contraction ; c'est entièrement que l'infini, quant à sa puissance, se dépouilla dans le fini, et lui confia ainsi son sort. Reste-t-il encore quelque chose, dès lors, pour une relation à Dieu ?" Et la réponse suit immédiatement, tout aussi claire que paradoxale : "Dieu, après s'être entièrement donné dans le monde en devenir, n'a plus rien à offrir : c'est maintenant à l'homme de lui donner ${ }^{41}$ ". Jonas fait alors appel aux « justes des nations », dont la " sainteté cachée est en mesure de compenser une faute innombrable, d'apurer le bilan d'une génération et de sauver la paix du Royaume invisible ${ }^{42}$ ». Voilà encore une idée principale qu'il énonçait déjà à propos du souci de Dieu : "Que Dieu porte le souci de ses créatures, voilà qui relève naturellement des principes les plus familiers de la foi juive. Mais notre mythe souligne un aspect moins familier, à savoir que ce Dieu soucieux n'est pas un magicien qui, par le seul acte de son souci, provoquerait simultanément la réalisation du but dont il a le souci : au contraire, il a laissé à d'autres acteurs quelque chose à faire, de sorte que son souci dépend d'eux ${ }^{43}$ ». On reconnaît ici le philosophe de la responsabilité humaine. Et il s'agit là, pour Jonas, non pas seulement d'un principe éthique, humanitaire ; la responsabilité comporte manifestement pour lui des connotations religieuses, liées au mandat de la création.

L'autre question qui doit nous retenir est celle du rapport de cette interprétation avec la foi chrétienne. Quand un chrétien lit, dans le mythe de Jonas, que Dieu " a renoncé à son Être propre ", qu'il " s'est dépouillé de sa divinité ${ }^{44}$ ", il ne peut s'empêcher de penser à la kénose du Christ, d'après Philippiens 2, 6 : "Lui étant dans la forme de Dieu n'a pas usé de son droit d'être traité comme un dieu, mais il s'est dépouillé prenant la forme d'esclave. » On a vu, en effet, que la théologie chrétienne du Dieu souffrant s'est élaborée en transposant sur la personne du Père l'idée de la kénose développée pour rendre compte de l'abaissement du Christ. Mais il y a aussi d'autres traits qui, chez Jonas, rappellent la figure du Christ. Par exemple, "le mystère des "trente-six justes" inconnus, qui d'après la doctrine juive ne doivent jamais manquer au monde pour sa continuation » fait penser au Christ, le juste par excellence, qui assure la rédemption du monde. Et

41. Ibid., p. 38.

42. Ibid., p. 39.

43. Ibid., p. 26-27.

44. Ibid., p. 15. 
la finale de la conférence introduit une idée plus étonnante encore à propos de la question de Job :

Ma réponse à moi s'oppose à celle du livre de Job : cette dernière invoque la plénitude de puissance du Dieu créateur, la mienne son renoncement à la puissance. Et pourtant - étrange à dire - toutes deux sont louange : car le renoncement se fit pour que nous puissions être. Même cela, me semble-t-il, est une réponse à Job : à savoir qu'en lui, Dieu même souffre ${ }^{45}$.

L'idée de l'autorenoncement est poussée ici à son extrême limite. Dieu se présente désormais comme faible et souffrant dans le monde, identifiant sa cause à celle des petits, des pauvres et des opprimés, souffrant avec eux et en eux. De toute évidence, nous nous retrouvons ici en plein évangile chrétien : le Christ considéré comme la nouvelle figure de Job, en qui Dieu souffre et meurt.

Cet arrimage avec la pensée chrétienne permet aussi de prolonger la théologie de Jonas, en lui apportant certains compléments essentiels. C'est ainsi qu'on pourrait, dans cette perspective, entrevoir ce qu'il advient de la toute-puissance divine. Du fait de l'auto-abaissement de Dieu, celle-ci n'est pas totalement perdue, comme le suppose la thèse de la mort de Dieu. Elle se retrouve plutôt sous une autre forme, dans la forme de l'immanence précisément. La toute-puissance apparaît alors au cœur même de la faiblesse et de la souffrance humaine ; et, dans l'évangile chrétien, son effet a pour nom la " résurrection ». D'ailleurs, dans ce même passage de Philippiens 2, 6-11, à l'idée de la kénose et de l'abaissement correspond celle de l'élévation et de la glorification. De plus, Hans Honas laisse entendre - bien discrètement il est vrai - qu'il pourrait y avoir, à la fin des temps, un retour de Dieu à la gloire de sa toute-puissance originelle. Quand il dit, par exemple, que Dieu "s'est dépouillé de sa divinité, afin d'obtenir celle-ci, en retour, de l'odyssée des temps ${ }^{46} »$. Et plus clairement encore, vers la fin de la conférence, quand il propose "l'idée d'un Dieu qui pour un temps - le temps que dure le processus continué du monde - s'est dépouillé de tout pouvoir d'immixtion dans le cours physique des choses de ce monde ${ }^{47}$ ».

45. Ibid., p. 39-40.

46. Ibid., p. 15.

47. Ibid., p. 34-35. 
Il reste que Jonas lui-même récuse tout rapprochement avec la pensée chrétienne d'un Dieu souffrant :

J'ai parlé d'un dieu souffrant - ce qui semble se trouver en contradiction directe avec la représentation biblique de la majesté divine. Naturellement, il y a le sens chrétien de l'expression " dieu souffrant ", mais mon mythe n'a pas à être confondu avec cela : il ne parle pas, comme cette formule le fait, d'un acte unique, par lequel la divinité, à un moment déterminé, et dans le but particulier de racheter l'homme, envoya une partie d'elle-même dans une situation de souffrance déterminée (l'incarnation et la crucifixion). Si quelque chose de ce que j'ai dit a un sens, c'est alors le suivant : à savoir que la relation de Dieu au monde implique une souffrance du côté de Dieu dès l'instant de la création ${ }^{48}$.

Ce que veut dire Jonas est assez clair. Selon la foi chrétienne, telle qu'il l'entend, Dieu ne se trouve pas totalement immergé dans le monde. Il demeure dans sa transcendance céleste d'où il peut intervenir à l'occasion, par exemple dans le miracle de la résurrection, pour sauver son Fils de la mort. Mais notons bien que, ce faisant, Jonas se réfère à un courant déterminé de la pensée chrétienne contemporaine, précisément celui qui refuse l'idée d'une kénose de Dieu. Dans une note au texte de Jonas, Catherine Chalier, théologienne juive elle-même, laisse entendre que la pensée chrétienne ne se limite pas à ce point de vue : "L'interprétation par H. Jonas peut être discutée, car la théologie chrétienne ne limite pas ainsi cette souffrance à un moment déterminé ${ }^{49}$ ». La question demeure donc ouverte quant à l'autre interprétation, celle que présentent tout spécialement les lettres de Bonhoeffer.

Or justement, dans son commentaire de la conférence de Jonas, Catherine Chalier fait coup sur coup trois références implicites à Bonhoeffer. Quand elle affirme : "La mémoire de ceux, juifs et non juifs, qui résistèrent spirituellement au désastre de la Choa, engage en effet à penser Dieu sans le confondre avec un Deus ex machina qui défend le faible contre le fort ${ }^{50}$ ». Quand elle ajoute que « cette impuissance [de

48. Ibid., p. 21-22.

49. Ibid., p. 41-42, note 3.

50. Catherine CHALIER, "Dieu sans puissance", dans H. JONAS, Le Concept de Dieu après Auschwitz, p. 63. - Cf. D. BONHOEFFER, Résistance et soumission. Lettres et notes de captivité, p. 290 (30 avril 1944) : Les gens religieux parlent de Dieu quand les connaissances humaines (quelquefois par 
Dieu] ne s'identifie à une pure et simple inexistence que dans l'esprit de ceux qui cherchent Dieu aux limites, toujours plus lointaines, de leur propre puissance, intellectuelle ou pratique, et non au cœur même de la $v_{i e}^{51} »$. Et quand elle conclut que « le Dieu qui, selon la tradition cabaliste surtout, est avec les hommes ne ressemble pas à une puissance qui maîtrise le cours des choses. Ce Dieu leur a dit qu'ils doivent apprendre à vivre sans cette hypothèse rassurante mais infantile ${ }^{52}$ ». Nous retrouvons donc Bonhoeffer au terme de notre cheminement, ce qui montre la

paresse) se heurtent à leurs limites ou quand les forces humaines font défaut c'est au fond toujours un deus ex machina qu'ils font apparaitre, ou bien pour résoudre apparemment des problèmes insolubles, ou bien pour le faire intervenir comme la force capable de subvenir à l'impuissance humaine ; bref, ils exploitent toujours la faiblesse et les limites des hommes. " — Voir encore ibid., p. 367 (16 juillet 1944) : "Voilà la différence décisive d'avec toutes les autres religions. La religiosité de l'homme le renvoie dans sa misère à la puissance de Dieu dans le monde, Dieu est le deus ex machina. La Bible le renvoie à la souffrance et la faiblesse de Dieu ; seul le Dieu souffrant peut aider. »

51. C. Chalier, dans Le Concept de Dieu après Auschwitz, p. 63. - Cf. D. BONHOEFFER, Résistance et soumission, p. 322 (29 mai 1944) : "Nous avons à trouver Dieu dans ce que nous connaissons et non pas dans ce que nous ignorons. Dieu veut être compris par nous non dans les questions sans réponse, mais dans celles qui sont résolues. Ceci est valable pour la relation de Dieu et la connaissance scientifique, mais également pour les problèmes simplement humains de la mort, de la souffrance et de la faute. Aujourd'hui, il existe des réponses humaines à ces questions qui peuvent faire abstraction de Dieu. En fait - et ç'a été ainsi de tout temps - les hommes arrivent à résoudre ces questions sans Dieu et il est faux de prétendre que le christianisme seul en connaît la solution. Les réponses chrétiennes ne sont ni plus ni moins convaincantes que d'autres solutions possibles. Ici non plus, Dieu n'est pas bouche-trou ; il doit être reconnu non à la limite de nos possibilités, mais au centre de notre vie. Dieu veut être reconnu non dans la mort seulement, mais dans la vie, dans la force et la santé et non seulement dans la souffrance, dans l'action et non seulement dans le péché. »

52. C. CHAlIER, dans Le Concept de Dieu après Auschwitz, p. 65. - Cf. D. BONHOEFFER, Résistance et soumission, p. 336 (8 juin 1944) : "L'homme a appris à venir à bout de toutes les questions importantes sans faire appel à "l'hypothèse Dieu". Cela va de soi dans les questions scientifiques, artistiques et même éthiques, et personne n'en doute ; depuis environ cent ans, ceci est de plus en plus valable pour les questions religieuses elles-mêmes ; il apparaît 
convergence d'une pensée juive et d'une pensée chrétienne qui, au coeur d'une même situation tragique, reconsidèrent leur foi en Dieu.

\section{Conclusions}

1. Le déplacement de la conscience chrétienne qui va du Pantocrator au Crucifié marque bien la situation religieuse présente. Une affirmation purement positive et naïve de la toute-puissance divine n'est plus possible hors de l'expression religieuse immédiate de la foi. Dès qu'intervient la réflexion théologique, on sent le besoin d'introduire des précisions, des nuances, des distinctions. Le Dieu tout-puissant ne peut plus être considéré comme quelqu'un qui peut faire tout ce qu'il veut. L'affirmation du Pantocrator doit être purifiée de toute connotation impériale. En somme, la confession de la toute-puissance ne peut plus se faire en dehors de son contexte judéo-chrétien. Ce contexte apporte alors une rectification critique au texte même du premier article du Credo chrétien.

Intervient donc ici un premier élément de réflexion critique. Et cette première critique de l'idée de toute-puissance est de nature biblique. Les Écritures juives aussi bien que chrétiennes présentent, en effet, une nouvelle image du Dieu tout-puissant, fortement modifiée par le contexte de l'alliance et de l'histoire du salut. C'est là-dessus qu'insiste Jean-Pierre Batut. D'autres études vont dans le même sens, parmi lesquelles je dois signaler le récent ouvrage d'Étienne Babut, au titre éloquent : Le Dieu puissamment faible de la Bible. L'auteur, pasteur de l'Église réformée, ne craint pas les paradoxes, on le voit bien. Il se réclame ouvertement de Bonhoeffer ; il inclut Hans Jonas dans sa bibliographie et il semble bien partager son point de vue, si l'on en

que tout va sans "Dieu" aussi bien qu'auparavant. Tout comme dans le domaine scientifique, "Dieu", dans le domaine humain, est repoussé toujours plus loin hors de la vie ; il perd du terrain. » - Voir aussi ibid., p. 366-367 (16 juillet 1944) : " Le Dieu qui nous laisse vivre dans le monde, sans l'hypothèse de travail Dieu, est celui devant qui nous nous tenons constamment. Devant Dieu et avec Dieu, nous vivons sans Dieu. Dieu se laisse déloger du monde et clouer sur la croix. Dieu est impuissant et faible dans le monde, et ainsi seulement il est avec nous et nous aide. Matthieu 8, 17 indique clairement que le Christ ne nous aide pas par sa toute-puissance, mais par sa faiblesse et ses souffrances. » 
croit sa conclusion intitulée : "Libérer Dieu du masque de la toutepuissance ${ }^{53}$. Il reste que Babut ne prétend pas faire autre chose que retracer l'image biblique de Dieu.

2. Il en va tout autrement de Hans Jonas lui-même. Bien sûr, celuici se situe résolument dans la tradition juive et il tient à montrer la continuité, la communauté d'esprit qui le relie aux auteurs bibliques. Mais il fait voir tout aussi clairement les ruptures qu'il se doit d'opérer. Dès le départ, il propose un nouveau mythe de la création, bien différent des récits de la Genèse. Et sa conclusion n'est pas moins radicale : "Ma réponse à moi s'oppose celle du livre de Job : cette dernière invoque la plénitude de la puissance du Dieu créateur, la mienne son renoncement à la puissance ${ }^{54}$ ». Tel est précisément le point crucial qui divise les positions. Le Dieu de l'alliance biblique bouleverse toutes les images reçues de la divinité, mais il reste transcendant, il demeure "Seigneur de l'histoire ». Pour sa part, Hans Jonas propose l'image d'un Dieu dépouillé de toute transcendance, totalement immanent au monde. Un Dieu si différent que plusieurs croyants ne le reconnaîtront plus.

D'où peut bien venir cette idée ? Jonas répond tout aussi directement et clairement : " Je propose, pour des raisons inspirées par l'expérience contemporaine de façon déterminante, l'idée d'un Dieu qui [...] s'est dépouillé de tout pouvoir d'immixtion dans le cours physique des choses de ce monde ${ }^{55}$ ». Toute la différence vient donc de la situation d'où il parle. Son concept de Dieu vient directement d'Auschwitz. Voilà bien une théologie qui accorde toute son importance au pôle de la situation. Celle-ci n'est plus simplement le lieu — ou le langage — où se trouve reçu et traduit le message de la révélation. C'est l'événement, le remous des eaux profondes où s'engouffre la foi, où elle meurt pour reprendre vie et réapparaître sous une forme nouvelle.

Tillich, dans sa Théologie systématique, a bien formulé cette corrélation du message de la révélation et de la situation historique. Mais il est loin d'en avoir montré toute la portée révolutionnaire, même s'il le laisse soupçonner avec des concepts comme "Ultimate Concern »

53. Étienne BABUT, Le Dieu puissamment faible de la Bible (lire la Bible, 118), Paris, Éditions du Cerf, 1999, p. 135-137.

54. H. JONAS, Le Concept de Dieu après Auschwitz, p. 39.

55. Ibid., p. 34-35. 
et « Ground of Being » (une expression reprise par Jonas au début de son mythe). Troeltsch est beaucoup plus clair là-dessus. Il ne craint pas d'affirmer la rupture tout autant que la continuité de sa théologie avec celle de la Bible : "L'importance de la Bible vient du fait qu'elle contient les premiers documents historiques. Le présent va toujours mesurer ses valeurs à son aulne, mais elle ne sera plus considérée comme "inspirée". La Bible ne sera donc plus la limite indépassable du christianisme. Elle va seulement conduire et féconder un développement qui va lui-même mener bien au delà 56 ». Troeltsch peut donc avouer que "le christianisme d'aujourd'hui n'est plus celui de la Bible $^{57}$ ", même s'il s'enracine toujours en elle. Voilà bien jusqu'où conduit la corrélation du message et de la situation. La conférence de Hans Jonas me semble la plus belle illustration de ce type de théologie.

3. La lecture de Hans Jonas nous invite enfin à considérer les apports mutels de la pensée juive et de la pensée chrétienne sur cette question de la kénose de Dieu. Je serais porté à dire ici que le texte de Jonas oblige la foi chrétienne à ouvrir ses horizons dans le sens de la protologie (la doctrine de la création), tandis que le dogme chrétien force le mythe de Jonas à se prolonger dans le sens de l'eschatologie (la doctrine du salut final).

Jonas interpelle la théologie chrétienne quand il constate qu'elle limite la souffrance de Dieu à un moment particulier de l'histoire, celui de la vie du Christ, alors que son mythe "implique une souffrance du côté de Dieu dès l'instant de la création ${ }^{58}$ ». Là-dessus Catherine Chalier apportait une nuance en citant Pascal (Pensées 736) : "Jésus sera en agonie jusqu'à la fin du monde; il ne faut pas dormir pendant ce temps-là ${ }^{59}$ ". Mais on pourrait prolonger encore, dans le sens de la protologie, en disant que Jésus est en agonie depuis le début du monde. Telle est précisément la thèse de Gérard Siegwalt : "Il y a une theologia crucis non seulement depuis Golgotha ou, d'une manière plus générale, depuis l'incarnation du Logos, mais depuis le commencement du monde, depuis qu'il y a des hommes qui ne reçoi-

56. E. Troeltlsch, Glaubenslehre (1925), Scientia Verlag Aalen, 1981, p. $9-10$.

57. Ibid., p. 27 : " das heutige Christentum ist nicht dasjenige der Bibel. »

58. H. JONAS, Le Concept de Dieu après Auschwitz, p. 22.

59. Ibid., p. 42, note 3. 
vent pas la lumière qui est celle de la vie, principe de toutes choses ${ }^{60}$ ". Cette thèse " que le Logos rédempteur est crucifié depuis l'origine ", Siegwalt l'appuie sur Apocalypse 13, 8 dans son énoncé littéral, où il est parlé de "l'agneau immolé dès la fondation du monde ${ }^{61}$ ». Cela concerne directement notre question du rapport entre le premier et le deuxième article du Credo. On ne peut plus l'entendre comme l'expression de deux moments historiques différents dans les relations entre Dieu et l'humanité. C'est du rapport intrinsèque entre la création et la rédemption du monde dont il s'agit. Et comme nous y invite encore Siegwalt dans son récent traité de la création, on doit le comprendre comme l'affirmation dialectique d'un unique mystère divin, dans son double aspect de transcendance et d'immanence : "il faut affirmer à la fois la permanence de Dieu par-delà l'impermanence du réel créé et l'advenir de Dieu dans sa création - sa présence à sa création - qui donne à celle-ci sa consistance ${ }^{62}$ » .

Nous avons vu par ailleurs que la foi chrétienne en la résurrection et au retour glorieux du Christ permettait de prolonger la pensée de Jonas en lui apportant certains compléments essentiels : la présence active de la toute-puissance au cœur de l'immanence souffrante sous la forme d'une victoire sur la mort (la résurrection), ainsi qu'un retour final (eschatologique) de Dieu à la gloire de sa toute-puissance originelle. Mais la question se pose alors : la croyance à la résurrection et au Royaume final est-elle compatible avec la thèse d'une totale immanence de Dieu, ou bien suppose-t-elle - pour utiliser le langage de Jonas - une partie de Dieu " préservée, immunisée, en état de diriger, de corriger, finalement de garantir depuis l'au-delà l'oblique formation de son destin au sein de la création ${ }^{63}$ »?

Bonhoeffer a bien perçu la difficulté : "On prétend qu'il est décisif que, dans le christianisme, l'espérance de la résurrection soit annoncée et qu'ainsi naisse une véritable religion de la rédemption. Tout le poids est donc sur l'au-delà de la mort. [...] Je le conteste.

60. Gérard SIEGWALt, Dogmatique pour la catholicité évangélique, I/1, Paris/Genève, Cerf/Labor et Fides, 1986, p. 89.

61. Ibid., p. 90.

62. G. SIEGWALT, Dogmatique pour la catholicité évangélique, III/2, Paris/ Genève, Cerf/Labor et Fides, 2000, p. 397.

63. H. JONAS, Le Concept de Dieu après Auschwitz, p. 14. 
L'espérance chrétienne de la résurrection se distingue en ceci de l'espérance mythologique qu'elle renvoie l'homme, d'une manière toute nouvelle et plus pressante que l'Ancien Testament, à la vie sur la terre $^{64}$ ». En somme, toute la question est de savoir de quelle transcendance il s'agit quand on parle de résurrection. Là encore, Bonhoeffer est bien clair quand il exclut toute transcendance de type supranaturaliste : "La foi en la résurrection n'est pas la solution du problème de la mort. "L'au-delà" de Dieu n'est pas l'au-delà de notre entendement. La transcendance théoriquement perceptible n'a rien de commun avec celle de Dieu. Dieu est au centre de notre vie tout en étant au-delà ${ }^{65} »$.

Bonhoeffer parle dans et à partir de la même situation que Jonas. Son interprétation de la foi chrétienne à la résurrection semble pleinement conforme au principe d'immanence tel que l'entend ce dernier. Avec cette différence toutefois que Jonas marque bien la rupture avec les croyances juives traditionnelles, tandis que Bonhoeffer laisse entendre qu'il ne fait que retrouver le sens initial de l'évangile de la résurrection. Il importe de clarifier la question en terminant. L'affirmation de la totale immanence de Dieu dans la création, le refus de toute transcendance supranaturaliste, ne compromet aucunement la foi au mystère de la résurrection, en tant qu'expression d'une transcendance divine au cœur même de l'immanence. Mais, de toute évidence, cela exclut la croyance au miracle de la résurrection corporelle, une telle croyance supposant nécessairement le maintien d'un Dieu transcendant, immunisé contre toute atteinte du devenir cosmique et de la souffrance humaine. Or il ne fait pas de doute que telle était bien la croyance des premières générations chrétiennes. On comprend dès lors que les chrétiens soient aujourd'hui divisés sur cette question du Pantocrator et du Crucifié, plusieurs refusant encore d'admettre une véritable kénose de Dieu.

64. D. BONHOEFFER, Résistance et soumission. Lettres et notes de captivité, p. 347-348 (27 juin 1944).

65. Ibid., p. 290-291 (30 avril 1944). 


\section{RÉSUMÉ}

Sur la question des rapports entre le Pantocrator (le premier article des anciens Symboles chrétiens) et le Crucifié (le deuxième article), nous présentons d'abord deux options bien différentes de théologiens contemporains : l'une qui souscrit à la thèse d'une "kénose » de Dieu (le Dieu crucifié), l'autre qui la refuse. Nous proposons ensuite nos propres vues, en suivant la ligne de pensée de Hans Jonas dans son «Concept de Dieu après Auschwitz ». Finalement, nous indiquons ce que la croyance chrétienne à la résurrection peut apporter à la thèse de Jonas et, réciproquement, comment la critique radicale de ce dernier peut aider à concevoir une foi non supranaturaliste en la résurrection.

\section{ABSTRACT}

On the question of the relationship between the Pantocrator (the first article of the ancient Christian creeds) and the Crucified (the second article), we first present here two different visions of contemporary Christian theologians: one which agrees with the idea of God's kenosis (the crucified God), the other which refuses it. Then we set forth our own position with the line of thought of Hans Jonas' concept of God after Auschwitz. Finally, we indicate how the Christian belief in the resurrection may help to further Jonas' views, while Jonas' sharp criticism should help Christians to conceive a non supranaturalist faith in the resurrection. 